\title{
VCAM-1 directed target-sensitive liposomes carrying CCR2 antagonists bind to activated endothelium and reduce adhesion and transmigration of monocytes
}

Calin, Manuela ; Stan, Daniela ; Schlesinger, Martin ; Simion, Viorel ; Deleanu, M ; Constantinescu, C A ; Gan, A M ; Pirvulescu, M M ; Butoi, E ; Manduteanu, I ; Bota, M ; Enachescu, M ; Borsig, Lubor ; Bendas, Gerd ; Simionescu, Maya

\begin{abstract}
Chemokines are critically involved in the development of chronic inflammatory-associated diseases such as atherosclerosis. We hypothesised that targeted delivery of compounds to the surface of activated endothelial cells (EC) interferes with chemokine/receptor interaction and thereby efficiently blocks inflammation. We developed PEGylated target-sensitive liposomes (TSL) encapsulating a CCR2 antagonist (Teijin compound 1) coupled with a specific peptide recognized by endothelial VCAM-1 (VpTSL-Tj). TSL were characterized for size (by dynamic light scattering), the amount of peptide coupled at the surface of liposomes and Teijin release (by HPLC). We report that Vp-TSL-Tj binds specifically to activated EC in vitro and in vivo, release the entrapped Teijin and prevent the transmigration of monocytes through activated EC. This is the first evidence that nanocarriers transporting and releasing chemokine inhibitors at specific pathological sites reduce the chemokine-dependent inflammatory process.
\end{abstract}

DOI: https://doi.org/10.1016/j.ejpb.2014.11.016

Posted at the Zurich Open Repository and Archive, University of Zurich

ZORA URL: https://doi.org/10.5167/uzh-118076

Journal Article

Accepted Version

Originally published at:

Calin, Manuela; Stan, Daniela; Schlesinger, Martin; Simion, Viorel; Deleanu, M; Constantinescu, C A; Gan, A M; Pirvulescu, M M; Butoi, E; Manduteanu, I; Bota, M; Enachescu, M; Borsig, Lubor; Bendas, Gerd; Simionescu, Maya (2015). VCAM-1 directed target-sensitive liposomes carrying CCR2 antagonists bind to activated endothelium and reduce adhesion and transmigration of monocytes. European Journal of Pharmaceutics and Biopharmaceutics, 89:18-29.

DOI: https://doi.org/10.1016/j.ejpb.2014.11.016 
VCAM-1 directed target-sensitive liposomes carrying CCR2 antagonists bind to activated endothelium and reduce adhesion and transmigration of monocytes

Authors and affiliations:

Manuela Calin ${ }^{1}$, Daniela $\operatorname{Stan}^{1}$, Martin Schlesinger ${ }^{2}$, Viorel Simion ${ }^{1}$, Mariana Deleanu ${ }^{1}$, Cristina Ana Constantinescu ${ }^{1}$, Ana-Maria Gan ${ }^{1}$, Monica Madalina Pirvulescu ${ }^{1}$, Elena Butoi ${ }^{1}$, Ileana Manduteanu ${ }^{1}$, Marian Bota ${ }^{3}$, Marius Enachescu ${ }^{3}$, Lubor Borsig ${ }^{4}$, Gerd Bendas ${ }^{2}$, Maya Simionescu $^{1}$

${ }^{1}$ Institute of Cellular Biology and Pathology "N. Simionescu" of the Romanian Academy, Bucharest, Romania

${ }^{2}$ Department of Pharmacy, University of Bonn, Bonn, Germany

${ }^{3}$ Center for Surface Science and Nanotechnology, University "Politehnica" of Bucharest, Bucharest, Romania

${ }^{4}$ Institute of Physiology, University of Zürich, Zürich, Switzerland 


\begin{abstract}
:
Chemokines are critically involved in the development of chronic inflammatory-associated diseases such as atherosclerosis. We hypothesised that targeted delivery of compounds to the surface of activated endothelial cells (EC) interferes with chemokine/receptor interaction and thereby efficiently blocks inflammation. We developed PEGylated target-sensitive liposomes (TSL) encapsulating a CCR2 antagonist (Teijin compound 1) coupled with a specific peptide recognized by endothelial VCAM-1 (Vp-TSL-Tj). TSL were characterized for size (by dynamic light scattering), the amount of peptide coupled at the surface of liposomes and Teijin release (by HPLC). We report that Vp-TSL-Tj binds specifically to activated EC in vitro and in vivo, release the entrapped Teijin and prevent the transmigration of monocytes through activated EC. This is the first evidence that nanocarriers transporting and releasing chemokine inhibitors at specific pathological sites reduce the chemokine-dependent inflammatory process.
\end{abstract}

\title{
Keywords
}

CCR2 antagonist, endothelium, monocyte, target sensitive liposomes, VCAM-1

\begin{abstract}
Abbreviations:
CCR2, CC chemokine receptor 2; EC, endothelial cells; DOPE, 1,2-dioleoyl-sn-glycero-3phosphoethanolamine; DOPA, 1,2-dioleoyl-sn-glycero-3-phosphate; PEG-DSPE, 1,2-distearoylsn-glycero-3-phosphoetanolamine-N-[poly(ethylene glycol)-2000]; Mal-PEG-DSPE, 1,2distearoyl-sn-glycero-3-phosphoethanolamine-N-[maleimide (polyethylene glycol)-2000]; TSL,
\end{abstract}


target sensitive liposomes; Vp-TSL, VCAM-1 recognizing peptide coupled to target sensitive liposomes; Vp-TSL-Tj, VCAM-1 recognizing peptide coupled to target sensitive liposomes encapsulating Teijin, TSL-Tj (target sensitive liposomes encapsulating Teijin without VCAM-1 recognizing peptide coupled); Vp-CL, VCAM-1 recognizing peptide coupled to conventional liposomes; Vp-CL-Tj, VCAM-1 recognizing peptide coupled to conventional liposomes encapsulating Teijin

\section{Introduction}

Atherosclerotic plaque formation is an inflammatory process that is associated with expression of a specific repertoire of chemokines and cell adhesion molecules by endothelial cells (EC). ${ }^{1}$ Both factors contribute to leukocyte recruitment to the developing atheroma. Chemokines are small, highly specialized polypeptides that function as modulators of cellular traffic through their interactions with $\mathrm{G}$ protein-coupled receptors at a spatially defined site. ${ }^{2}$ Chemokines bind to glycosaminoglycans (GAGs) present on the EC membrane and guide leukocyte entry into the vessel wall. Based on the key importance of chemokines in the development of atherosclerosis, a pharmacological interference at the level of chemokines may open new avenues in the prevention and treatment of this disorder. Thus, functional manipulation of the chemokine system using chemokine antagonists (CA) or chemokine receptor antagonists (CRA) constitutes

an important therapeutic option to prevent the accumulation of inflammatory immune cells that drives atherogenesis. However, systemic administration of chemokine antagonists or interventions in the function of one or more chemokines have potentially a high risk of side 
effects, such as impaired host defence against pathogens. Experimental and clinical testing of potential inhibitors of chemokines and their receptors for the treatment of inflammatory diseases revealed detrimental immunological effects. ${ }^{3}$ Therefore, there is a need for the development of new and innovative therapeutic approaches or strategies to manipulate selectively specific chemokine(s) function in a particular pathophysiological context with few or no adverse effects on the immune system. We hypothesised that the local targeted delivery of CA or CRA at specific sites utilizing appropriately designed carriers may constitute a novel and improved strategy to hold back or interrupt a confined inflammatory process.

Despite progress in the field of targeted carrier technologies, different approaches require a design of carriers with specific properties with respect to tissue targeting. To interfere with the chemokine axis within the local microenvironment at the EC surface, the carrier system should have the potential to: i) efficiently entrap drugs (CA or CRA), ii) target sequences of specific receptors on the activated endothelial surface, and iii) have a specific release mechanism for the drug cargo at the site of adhesion prior to cellular uptake. In this respect, liposomes appear most promising thanked to their tunable properties in drug loading and release characteristics as well as their manifold coupling technologies for specific homing devices.

Vascular targeting of liposomes was previously applied in inflammatory-related diseases, usually combined with targeting EC adhesion receptors. ${ }^{4-8}$ The shortcoming of this approach is that the liposomes were predominantly taken up by EC, an effect entirely inadequate when inhibition of chemokine/chemokine receptor interaction at the cell's surface is sought after. ${ }^{9-11}$ To circumvent this problem, we revisited an old principle of target-triggered release of drugs from target-sensitive liposomes (TSL) initially described by Huang and colleagues. ${ }^{14-17}$ These carriers have a specific phospholipid bilayer composition, which upon target recognition and binding 
exhibit destabilization that is followed immediately by payload release. ${ }^{12-15}$ Taking advantage of these properties, we hypothesized that these type of liposomes modified accurately for our aim, could be employed successfully in the chemokine targeting approaches. Here we demonstrate that the principle of TSL combined with PEGylation leads to particles that characteristically have long storage and circulation time and concomitantly maintain the trigger-release properties. We provide evidence that PEGylated TSL coupled with a recognizing peptide directed specifically against vascular cell adhesion molecule-1 (VCAM-1) and encapsulating an antagonist to CCR2 namely Teijin compound $1,(\mathrm{Vp}-\mathrm{TSL}-\mathrm{Tj})$ bind specifically to activated EC in culture and inhibit the ensuing adhesion and transendothelial migration of monocytes. In ApoE-deficient mouse, Vp-TSL target specifically aortic plaque endothelial VCAM-1 and Teijin compound 1 reduces the mouse monocyte/macrophage cell line (RAW 264.7) adhesion/infiltration into the aorta. This is a novel and promising approach to target initial inflammatory process that is common critical event in numerous major diseases, including atherosclerosis.

\section{Materials and methods}

\subsection{Reagents}

Reagents were obtained from following sources: 1,2-dioleoyl-sn-glycero-3phosphoethanolamine (DOPE), 1,2-dioleoyl-sn-glycero-3-phosphate (sodium salt) (DOPA), 1,2distearoyl-sn-glycero-3-phosphoethanolamine-N-[maleimide (polyethylene glycol)-2000] (MalPEG-DSPE), 1,2-distearoyl-sn-glycero-3-phosphoetanolamine-N-[poly(ethylene glycol)-2000] (PEG-DSPE), 1,2-dipalmitoyl-sn-glycero-3-phosphoethanolamine-N-(lissamine rhodamine B 
sulfonyl) (ammonium salt) (Rhodamin-PE) and 1-palmitoyl-2-oleoyl-sn-glycero-3phosphocholine (POPC) from Avanti Polar Lipids (Alabaster, AL/USA), mouse anti-human VCAM-1, irrelevant mouse IgG1 and rhVCAM-1 Fc chimera from R\&D Systems (Biomedica Medizinprodukte Romania SRL), Dulbecco's modified Eagle's medium (DMEM), fetal calf serum (FCS) from Gibco BRL (Antisel Selidis Ro SRL), cell culture plates from Corning (New York, NY/USA), black 96-well plates from Nalge NUNC International (Rochester, USA), peptide with high affinity for VCAM-1 (VHPKQHRGGSKGC) was synthesized by GeneCust (Dudelange, Luxembourg), Amicon centrifugal filter columns with a cutoff of $100 \mathrm{kDa}$ from Millipore (Merk Romania SRL), transmigration chambers from Costar Europe Ltd. (Badhoevedorp, The Netherlands), tris (2-carboxyethyl)phosphine (TCEP), phospholipid determination, bicinchoninic acid protein assay kit, as well as all other chemicals were from Sigma-Aldrich (Redox Lab Supplies Com S.R.L.).

\subsection{Preparation of VCAM-1 directed, PEG-stabilized Target-Sensitive Liposomes (Vp-TSL)}

\subsubsection{Preparation of TSL}

PEG-stabilized TSL were prepared by a mixture of DOPE, DOPA and a functionalized phospholipidic anchor (Mal-PEG-DSPE) used to covalently couple the peptide with affinity for VCAM-1 to the liposome surface. As control, non-sensitive, conventional liposomes (CL) were prepared using POPC and cholesterol instead of DOPE and DOPA (POPC:Chol:Mal-PEGDSPE:DSPE-PEG at a molar ratio 60:35:2:3 mol\%). Unilamellar liposomes with different composition and lipid ratio (as indicated in supplementary table 1, please visit 
http://pubs.acs.org) were obtained by extruding multilamellar vesicles through polycarbonate membranes as previously described. ${ }^{16}$ Briefly, the mixture of phospholipids in chloroform was dried in a rotary evaporator under reduced pressure. The resulted lipid film was hydrated with a solution containing the substance to be encapsulated into liposomes, i.e. phosphate buffered saline (PBS), calcein or CCR2 antagonist, to reach the final lipid concentration of $10 \mu \mathrm{mol} / \mathrm{ml}$. The resulting multilamellar vesicles, obtained by thorough mixing the aqueous solutions of lipids, were extruded 10 times through a $100 \mathrm{~nm}$ polycarbonate membrane using a Mini-Extruder (Avanti Polar Lipids, Alabaster, AL/USA).

To fluorescently label the phospholipid bilayers of liposomes, 1 mol \% Rhodamin-PE was added as an ethanol solution subsequent to liposomes preparation.

For content release studies, the liposomes were prepared by hydration of the phospholipid mixture with a solution of $100 \mathrm{mM}$ calcein or CCR2 antagonist (Teijin compound 1). Removal of non-incorporated calcein or CCR2 antagonist was accomplished by gel filtration on a Sephadex G-50 column using as an eluting solution the following buffer: $10 \mathrm{mM} \mathrm{Na} 2 \mathrm{HPO}_{4}, 10$ $\mathrm{mM} \mathrm{NaH} 2 \mathrm{PO}_{4}, 2 \mathrm{mM}$ EDTA and $30 \mathrm{mM} \mathrm{NaCl}$, pH:6.7 (coupling buffer).

\subsubsection{Coupling of VCAM-1 recognizing peptide to the surface of TSL}

A VCAM-1 binding peptide with the amino acids sequence VHPKQHRGGSKGC (described by Kelly et al. ${ }^{17}$ ) has been coupled to the maleimide group at the distal end of PEG by sulfhydrylmaleimide as previously described. ${ }^{18}$ Before coupling, the peptide was reduced with Tris (2carboxyethyl) phosphine (TCEP) (2 hours at room temperature) that breaks the disulfide bond between peptides and activates the sulfhydryl group. The excess TCEP was removed by dialysis 
(using cellulose ester membrane with a cut-off of 500-1000 Da) against coupling buffer, overnight at $4^{\circ} \mathrm{C}$. Then, an aliquot of the solution of the VCAM-1 recognizing peptide in coupling buffer was added at a concentration of $10 \mu \mathrm{g}$ peptide/ $\mu$ mol total lipid to maleimide functionalized PEG-DSPE containing liposomes and incubated overnight at room temperature under shaking. To saturate non-reacted maleimide, $1 \mathrm{mM}$ L-cystein was added for 30 minutes at room temperature and the unbound peptide was removed employing centrifugal filter columns with a cut off $100 \mathrm{kDa}$. Control liposomes (with no peptide coupled) were prepared from the same lipid mixture in which the reactive maleimide groups were blocked with L-cystein.

The amount of peptide coupled at the surface of liposomes was quantified by HPLC employing a UHPLC-Agilent 1290 Infinity with a Zorbax SB C18 2.1x100 mm, $1.8 \mu \mathrm{m}$ column and a gradient of $0.1 \%$ TFA in water (A) and $0.1 \%$ TFA in acetonitrile (B) (from 1\% B until 26\% B in A for 10 minutes).

\subsection{Vp-TSL characterization}

Liposomes prepared as described above were characterized for their physical properties (size, temperature and serum-stability) and functionality (binding to the target, content release).

\subsubsection{Size}

The size distribution and the average diameter of the resulting liposomes was determined by photon correlation spectroscopy using Nicomp submicron particle analyzer model 380 (Nicomp Inst Corp, Santa Barbara, CA) and the multi-modal distribution. The vesicle suspensions were 
diluted with fresh $(0.22 \mu \mathrm{m}$-filtrated) PBS in order to reach a count rate of 250 to $350 \mathrm{kHz}$. The instrument parameters were set as follows: automatic choice of channel width, vesicle mode, volume weighting and automatic change from Gaussian distribution mode to multimodal mode if the value of Chi-squared exceeded 3.00.

\subsubsection{Temperature-dependent stability of Vp-TSL}

The stability of Vp-TSL at $4^{\circ} \mathrm{C}$ (storage stability) and $37^{\circ} \mathrm{C}$ was tested by quantifying the leakage of the liposome-entrapped calcein from Vp-TSL prepared with different content of DOPE/DOPA. Calcein $(100 \mathrm{mM})$ was encapsulated into the liposomes; at this concentration calcein fluorescence is self-quenched and has thus a low value. The calcein released after storage at $4{ }^{\circ} \mathrm{C}$ for different time intervals (up to 50 days) and upon incubation at $37^{\circ} \mathrm{C}$ up to 24 hours was determined by measuring the increase in the fluorescence intensity with excitation and emission wavelengths of $490 \mathrm{~nm}$ and $520 \mathrm{~nm}$ with a spectrofluorimeter plate reader TECAN (using 96 well black plates). The percentage of calcein released was calculated according to the formula introduced by Ho et al. ${ }^{13}$ :

$\%$ calcein release $=(\mathrm{F}-\mathrm{F} 0) /(\mathrm{FTx}-\mathrm{F} 0) \times 100 \quad[1]$

where F0 represents the calcein fluorescence immediately after liposome preparation, $\mathrm{F}$ is the calcein fluorescence at a certain time point and FTx is the total fluorescence after adding $1 \%$ Triton X-100.

\subsubsection{Serum stability of Vp-TSL}


Calcein-loaded TSL with different PEG-lipids ratios were added to a 96-well black plate containing $20 \%$ or $50 \%$ serum in PBS and incubated at $37^{\circ} \mathrm{C}$ for 24 hours using a PHERAstar reader BMG Labtech equipped with a temperature controlled chamber. The increase in calcein fluorescence over time was measured and analysed using Mars software. The calcein release was determined using the above formula [1].

\subsection{Determination of binding of VCAM-1 directed-TSL (Vp-TSL) to activated endothelial cells}

\subsubsection{Endothelial cell culture}

Human endothelial cells (EC), EA.hy926 line (purchased from American Type Culture Collection) were cultured in Dulbecco's modified Eagle's medium (DMEM) supplemented with $10 \%$ fetal calf serum, $100 \mathrm{U}$ penicillin, $100 \mu \mathrm{g}$ streptomycin, $50 \mu \mathrm{g}$ neomycin $/ \mathrm{ml}, 100 \mu \mathrm{M}$ hypoxanthine and $16 \mu \mathrm{M}$ thymidine, at $37^{\circ} \mathrm{C}$ in a $5 \% \mathrm{CO}_{2}$ incubator as previously indicated. ${ }^{19}$

\subsubsection{VCAM-1 expression on activated EC membrane}

The expression of VCAM-1 on the surface of EC after stimulation for 24 hours with 10 $\mathrm{ng} / \mathrm{ml}$ TNF- $\alpha$ was determined by flow cytometry using the appropriate antibodies and a standard flow cytometry protocol.

\subsubsection{Binding of $\mathrm{Vp}-\mathrm{TSL}$ to activated endothelial cells}


Quiescent or TNF- $\alpha$ activated EC were fixed in 1\% PFA and incubated for $1 \mathrm{~h}$ at room temperature with $0.25 \mu \mathrm{mol} / \mathrm{ml}$ Rhodamine-PE-labelled targeted Vp-TSL or non-targeted TSL in $100 \mu 1$ incubation solution containing $1 \%$ BSA in PBS. After one hour, to remove unbound liposomes, the cell/liposome suspensions were washed three times with cold PBS and then analyzed by flow cytometry (Gallios Flow Cytometer from Beckman Coulter). The specific binding of Vp-TSL to activated EC was assessed by a competitive binding assay. Briefly, VpTSL were pre-incubated for 30 minutes with an excess of soluble VCAM-1 $(10 \mu \mathrm{g} / \mathrm{ml})$ and then incubated with EC. After 60 minutes the monolayers were washed three times to remove unbound liposomes and examined with an inverted fluorescence microscope (Olympus IX81).

\subsection{Evaluation of the specificity of binding of Vp-TSL to activated EC surface and their fate}

\subsubsection{Vp-TSL binding studies by Surface Acoustic Wave (SAW) sensors}

The liposomal binding onto VCAM-1 was investigated by the mass-sensitive technology of Surface Acoustic Wave sensors using a SAM blue ${ }^{\circledR}$ device (SAW Instruments GmbH, Bonn, Germany) as described before ${ }^{20}$ for Fc-tagged rh VCAM-1 immobilised on gold-coated sensors. The sensors were pre-incubated in an ethanol solution of 11-mercaptoundecanoic acid (10 mM) that formed a self-assembled monolayer. The terminal carboxyl groups were activated with a mixture of $200 \mathrm{mM} \mathrm{N}$-ethyl- $N$-(dimethylaminopropyl)-carbodiimide (EDC) and $50 \mathrm{mM} \mathrm{N}$ hydroxysuccinimide (NHS) to bind $50 \mu \mathrm{g} / \mathrm{mL}$ VCAM-1 Fc-chimera. Excess of free activated 
NHS esters was blocked by $1 \mathrm{mM}$ ethanolamine ( $\mathrm{pH} 8.5)$. After immobilisation of the proteins, a 15 min baseline equilibration in PBS was performed. The flow rate was adjusted to $40 \mu \mathrm{L} / \mathrm{min}$. For binding experiments, the liposomes were used in dilution series between $10^{-10} \mathrm{M}$ and $10^{-3} \mathrm{M}$ and binding events were measured as phase shifts of the acoustic wave.

\subsubsection{Atomic Force Microscopy}

For topographic investigations, similar gold sensors as for SAW-sensor measurements were utilized. The technique employed was non-contact Scanning Polarization Force Microscopy (SPFM) that uses the electrostatic interactions between a charged tip (kept at $20 \mathrm{~nm}$ distance from the sample) and the sample whose topography is to be investigated. The SPFM scans were performed in AC mode with $5 \mathrm{~V}$ amplitude bias applied between the tip and the sample at $3 \mathrm{kHz}$. The surfaces before and after hydration show no change in the topography. The microscope chamber had $\sim 60 \%$ humidity so that the water film on the surface of the sensor would not evaporate too quickly. After the surface has been initially scanned, the liposomes were added and the excess solution was removed by absorption with a tissue paper from the side without touching the investigated surface.

\subsubsection{Content release after binding of Vp-TSL to the activated EC surface}

Static conditions. Calcein was used as a model compound for these experiments. The kinetics of calcein released from TSL $(10 \mu \mathrm{M})$ endowed with 5 mol \% PEG upon incubation with quiescent or TNF- $\alpha$-activated EC (fixed in $1 \%$ paraformaldehyde) was followed for 1 to 24 
hours at $37^{\circ} \mathrm{C}$ by fluorescence measurements using a PHERAstar reader BMG Labtech equipped with a temperature controlled chamber. As control, conventional liposomes (CL) were used. The data obtained on the release in calcein fluorescence over time were analysed using Mars software and calculated using formula [1] described above.

Flow conditions. To mimic the in vivo conditions, we performed flow chamber experiments in which glass slides were covered with $0.2 \mu \mathrm{g}$ rhVCAM-1 Fc chimera or with cultured quiescent or TNFa-activated EC. The slides were incorporated into a parallel plate flow

chamber $^{5}$ that was fixed on an inverted fluorescence microscope, and the flow medium (PBS, pH 7.4) was driven at a shear rate of $\sim 200 \mathrm{~s}^{-1}$. Different types of various prepared liposomes were added to a total volume of $\sim 2 \mathrm{ml}$ and the marker released was determined at different time points within $24 \mathrm{~h}$. For this, $50 \mu \mathrm{L}$ aliquots were collected and the fluorescence was quantified with a POLARstar Galaxy plate reader (BMG Labtech).

\subsection{Entrapment of CCR2 antagonist-Teijin- into targeted TSL (Vp-TSL-Tj) and its effects}

\subsubsection{Teijin entrapment into TSL}

The CCR2 antagonist, Teijin compound 1: ( $N$-[2-[[(3R)-1-[(4-chlorophenyl)methyl]-3pyrrolidinyl] amino]-2-oxoethyl]-3-(trifluoromethyl)benzamide hydrochloride) was encapsulated into the aqueous phase of TSL by hydrating the lipid film with Teijin solution, at a drug:lipid ratio of 1:5, as described in section 2.2.1. The incorporation rate of Teijin was evaluated using UHPLC Agilent 1290 Infinity system with a column Zorbax SB C18 2.1x100 mm, $1.8 \mu \mathrm{m}$. The mobile phase was a mixture consisting of $43 \%$ acetonitrile and $57 \% 50 \mathrm{mM}$ ammonium acetate 
at $\mathrm{pH}$ 8. Detection of Teijin compound was done at $220 \mathrm{~nm}$ using a flow rate of $1 \mathrm{ml} / \mathrm{min}$ in isocratic mode at $25^{\circ} \mathrm{C}$.

\subsubsection{Teijin release from Vp-TSL-Tj after liposome binding to EC surface}

Activated cultured EC were incubated with Vp-TSL-Tj, non-targeted TSL (TSL-Tj) and VCAM1 recognizing peptide coupled to conventional liposomes encapsulating Teijin (Vp-CL-Tj) at 1 $\mu \mathrm{mol} / \mathrm{ml}$ and after defined times, the culture media were collected and the intact liposomes were separated from the released Teijin using the Millipore centrifugal filter units of $100 \mathrm{kDa}$ cut off. Teijin concentration was determined by HPLC as described above and the release was calculated in relation to the start concentration of Teijin in liposomes before incubation.

\subsubsection{Effect of Vp-TSL-Tj on human monocyte adhesion to human activated cultured EC}

Monocytes were isolated from human peripheral blood as described in supplementary material (please visit http://pubs.acs.org). The effect of Teijin compound 1 on monocyte adhesion to activated endothelium was monitored using EC seeded in 24-well plates and activated (24 hours) with $10 \mathrm{ng} / \mathrm{ml}$ TNF- $\alpha$. Human monocytes were fluorescently labelled with 2'7'-bis (2carboxyethyl)-5(6)-carboxy fluorescein acetoxymethyl ester (BCECF-AM) as previously

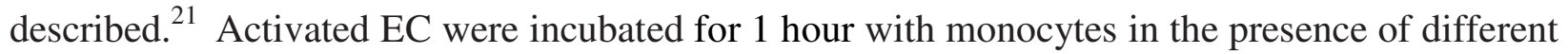
concentrations of Teijin either free or encapsulated into Vp-TSL-Tj, non-targeted TSL-Tj or into $\mathrm{Vp}-\mathrm{CL}-\mathrm{Tj}$. The number of adhered monocytes was determined using a fluorescence microscope Olympus IX81 and CellSense Dimensions software. The percent inhibition of monocyte 
adhesion to activated EC in the presence of free or liposome-encapsulated Teijin was calculated relative to control (monocytes incubated with activated EC).

\subsubsection{Effect of Vp-TSL-Tj on monocyte transmigration through activated EC monolayer}

The efficacy of chemokine receptor inhibition by Vp-TSL-Tj on transmigration of human monocytes through activated EC was assessed by classical transmigration assay using Boyden chambers. The EC were seeded on the insert's filter and exposed for 24 hours with $10 \mathrm{ng} / \mathrm{ml}$ TNF- $\alpha$. Activated EC were incubated with $100 \mu \mathrm{g} / \mathrm{ml}$ Teijin either free or encapsulated as VpTSL-Tj or TSL-Tj or Vp-CL-Tj in the presence of BCECF-AM labelled monocytes. After 16 hours, transmigration of monocytes was assessed using a serum gradient (1\% FCS in the upper chamber and $10 \%$ FCS in the lower chamber) and measuring the fluorescence of migrated monocytes attached to the filter as well as in the lower chamber. The percent inhibition of monocyte transmigration was determined relative to control (monocytes incubated with activated EC in the absence of free or Teijin-encapsulated liposomes).

\subsection{Animal studies}

\subsubsection{The animal models}

Male ApoE-/- mice (C57BL/6 genetic background) and C57BL/6 (wild type) mice from Charles River Laboratories were used. All animals had access to standard rodent diet, water ad libitum, and were kept in a temperature-controlled chamber at $24^{\circ} \mathrm{C}$ with a $12 \mathrm{~h}$ light/dark cycle. At seven 
weeks of age, ApoE-/- mice were fed with a high-fat, cholesterol-rich (1\%) diet for another six weeks. The experiments on mice had the approval of the Ethics Committee of our institution and were conducted in accordance with the EU guidelines, annexe to Directive 86/609, Appendix A of the European Convention for the protection of vertebrate animals used for experimental and other scientific purposes (ETS no. 123), Strasbourg, 2006.

\subsubsection{Binding of Vp-TSL-Tj to the aortic endothelium of ApoE-deficient mice}

These experiments were carried out by perfusing Vp-TSL or TSL (non-targeted liposomes) in situ in ApoE-/- mice kept for six weeks on Western-type diet, and as control on C57BL/6 mice, maintained on chow diet. The mice were anesthetized with ketamine/xylasine and then the vasculature was washed out of blood by injection into the left ventricle of PBS buffer using a peristaltic pump (infusion rate $2.5 \mathrm{ml} / \mathrm{min}$ ). This was followed by administration of fluorescently (Rhodamine-PE) labelled Vp-TSL and TSL at a concentration of $1 \mu \mathrm{mol} / \mathrm{ml}$. A gentle ligature placed on the abdominal aorta, allowed the close contact of liposomes with the vessel for 20 minutes, after which the ligature was removed and the vasculature extensively washed (10 minutes, $2.5 \mathrm{ml} \mathrm{PBS/min.)} \mathrm{to} \mathrm{dispose} \mathrm{of} \mathrm{liposomes} \mathrm{that} \mathrm{were} \mathrm{not} \mathrm{bound} \mathrm{to} \mathrm{the} \mathrm{vascular}$ endothelium. Then, the aortas were excised and the fluorescence was measured by an IVIS Imaging System 200 (Caliper Life Sciences) using $\lambda_{\mathrm{ex}}=535 \mathrm{~nm}$ and $\lambda_{\mathrm{em}}=620 \mathrm{~nm}$.

\subsubsection{Effect of Teijin on monocytes adhesion/infiltration into mouse aorta in situ}


The effect of CCR2 antagonist on monocytes adhesion and infiltration into the atherosclerotic plaque was assessed as previously described. ${ }^{22}$ Briefly, RAW 264.7 cells (mouse monocyte/macrophage cell line) which reportedly express CCR2 receptors ${ }^{23}$ were fluorescently labelled by incubation (18 hours) with Rhodamine-labelled conventional multilamellar liposomes ( $1 \mu \mathrm{mol}$ lipids $/ 10^{6}$ cells) and the cellular fluorescence was assessed by the fluorescence plate reader TECAN. Subsequently, labelled cells, pre-incubated or not with Teijin (40 $\mu \mathrm{g} / \mathrm{ml}$, for 30 minutes), were incubated in situ with the aortas of ApoE-deficient or C57BL/6

mice $\left(10 \times 10^{6}\right.$ cells/mouse) using the same procedure as described in section 2.7.2. After thorough washing of the vasculature, the fluorescently labelled cells adhering to-, or infiltrated into the aorta were visualised by the IVIS Imaging System $200(\lambda \mathrm{ex}=535 \mathrm{~nm} ; \lambda \mathrm{em}=620 \mathrm{~nm})$.

\section{8. Statistical analysis}

The results were expressed as mean \pm S.E.M. and all the experiments were performed at least in triplicates. Statistical evaluation was carried out by one-way analysis of variance between groups (ANOVA program of Origin). Differences were considered to be statistically significant when $\mathrm{p}<0.05$.

\section{Results}

\subsection{Preparation and characterization of VCAM-1 targeted TSL (Vp-TSL)}


We prepared PEG-stabilized TSL consisting of a balanced bilayer mixture of DOPE, DOPA and a PEGylated phospholipid used for coupling of homing devices. A series of liposomes that varied slightly in the lipid composition were obtained by varying fractions of DOPA or the PEG derivative, as outlined in supplementary Table 1 (please visit http://pubs.acs.org).

For targeting the activated vascular endothelium we choose the surface exposed protein VCAM-1 that reportedly is considered the most promising target, characteristically expressed under inflammatory conditions. ${ }^{4,24,25}$ A peptide with affinity for VCAM-1 was covalently coupled via maleimide to the distal end of functionalized PEG-phospholipids incorporated into liposomal bilayers. HPLC results showed no detectable free peptide in the liposome preparations indicating a coupling efficiency of $\sim 10 \mu \mathrm{g}$ peptide/ $\mu \mathrm{mol}$ phospholipid. There were no significant differences between peptide-coupled TSL (Vp-TSL) and non-targeted TSL with respect to the average hydrodynamic diameter (immediately after preparation or during storing periods) that was around $130 \mathrm{~nm}(127.58 \pm 18.88 \mathrm{~nm}, \mathrm{n}=8)$ and had a unimodal size distribution (as shown by the polydispersity index) always smaller than 0.2 .

The entrapment stability of the different liposomal preparations was determined following calcein release as a function of time, temperature, storage in PBS or PBS supplemented with serum. We determined the release of calcein from TSL composed of 5 mol \% PEG and different concentration of DOPA in the phospholipid bilayer (supplementary Table 1, please visit http://pubs.acs.org) after incubation in buffer (at $4^{\circ} \mathrm{C}$ ) for time intervals up to 50 days (Figure 1A) and for up to 24 hours at $37^{\circ} \mathrm{C}$ (Figure 1B). The experiments showed that the lowest calcein release was determined for Vp-TSL with 35\% DOPA content; at this concentration, no noticeable increase of calcein released within 50 days of storage at $4^{\circ} \mathrm{C}$ was detected. Moreover, the release of calcein from Vp-TSL with $35 \%$ DOPA content at $37^{\circ} \mathrm{C}$ was $\sim 20 \%$, whereas other 
formulation such as TSL with 10 mol \% DOPA released more than $70 \%$ calcein in the same conditions (24 hours).

It is accepted that a certain fraction of PEG-PE is essential to avoid destabilization and to provide sufficient stability of liposomes in a serum environment. Conversely, PEG-PE antagonizes the target-sensitive release mechanism. Thus, to investigate the relation between PEG-concentration and serum stability of the prepared liposomes, calcein release from TSL with different content of PEG-lipids was evaluated at $37^{\circ} \mathrm{C}$ in PBS containing $20 \%$ or $50 \%$ serum. Figure $1 \mathrm{C}$ and D summarize the percent release of calcein from TSL (DOPE: DOPA: PEG-DSPE: Mal-PEGDSPE) composed of $35 \mathrm{~mol} \%$ DOPA and different concentration of phospholipid derivatives of PEG [from 1 to $5 \mathrm{~mol} \%$ PEG, please see also supplementary Table 1 available at http://pubs.acs.org)

We observed that the percent of calcein released after incubation of TSL in $20 \%$ serum decreased as the concentration of PEG-lipids into bilayers increased. Thus, a burst release of calcein $(\sim 65 \%)$ was detected after 1 hour incubation in serum of TSL containing 1 mol \% PEG, whereas in the case of TSL with 5 mol \% PEG the percent release was $10 \%$. After 24 hours, TSL with 1 mol \% PEG released over $95 \%$ of encapsulated calcein while for TSL with 5 mol \% PEG the calcein released was $\sim 60 \%$. Incubation of TSL with $50 \%$ serum determined a high destabilization of TSL causing at 1 hour a release of calcein from TSL with 1 mol \% PEG of $\sim 75 \%$ and from TSL with 5 mol \% PEG of $\sim 40 \%$. At 24 hours, the release of entrapped calcein from TSL with $1 \mathrm{~mol} \%$ PEG was $80 \%$ and from TSL with 5 mol \% PEG, $70 \%$. 
Based on the above results on the stability of stored TSL in buffer and in sera, for all further functional studies of targeted release, the nanoparticles having in composition $35 \mathrm{~mol} \%$ DOPA and 5 mol \% PEG were employed.

\subsection{VCAM-1 directed TSL (Vp-TSL) target specifically activated endothelial cells}

The specificity of binding of Vp-TSL to activated EC was investigated by flow cytometry and fluorescence microscopy. First, we assessed and confirmed by flow cytometry that as expected, in our experimental conditions TNF- $\alpha$-activated EC express VCAM-1 on their surface (supplementary Figure 1). The experiments performed demonstrated that non-targeted TSL do not recognize or bind to a very low level to activated EC $(<1 \%)$. In contrast, as shown in Figure 2A, Vp-TSL bound specifically to activated EC and the binding was significantly higher compared to the binding to non-activated EC (40\% versus $\sim 10 \%$, respectively). The specific binding of Vp-TSL to activated EC (Figure 2B) was completely blocked by pre-incubation of Vp-TSL with an excess of soluble recombinant VCAM-1 $(10 \mu \mathrm{g} / \mathrm{ml})$ prior to exposure to the activated EC (Figure 2C). Together these data indicated clearly the specificity of Vp-TSL to TNF- $\alpha$ activated EC.

\subsection{Fate of Vp-TSL after the specific binding to endothelial VCAM-1}

The triggered release of a drug payload from TSL at the target site is likely induced by a liposomal fusion with the target surface or by an aggregation process inducing massive liposomal destruction. In order to investigate whether the TSL selected above show such as 
destruction, we followed the fate of liposomes after binding to a simulated target surface applying either a biosensor approach or atomic force microscopy.

The liposome binding and its fate after binding onto a VCAM-1 covered sensor was investigated by mass-sensitive technology of Surface Acoustic Wave. It became evident from the phase shifts that Vp-TSL injected in increasing concentrations, bind to the target protein (Figure 3A) but not to the plain TSL lacking the peptide (Figure 3C). Most interestingly, Vp-TSL induce a certain increase in oscillation amplitude after the initial binding, which indicated a decreased surface viscoelasticity due to an intensive surface contact of the liposomes, most likely a flattening or fusion at the target surface (arrow in Figure 3B). This effect was not achieved when non-targeted TSL or VCAM-1 targeted liposomes of conventional lipid composition were tested. The results strongly suggested the flattening or fusion of the Vp-TSL at the target surface.

Using Scanning Polarization Force Microscopy (SPFM) we obtained scans as 2D and 3D images of VCAM-1 covered gold sensor surface subsequent to the addition of Vp-TSL (Figure 3D and insert). The liposomes appeared as structural features of 150-250 nm diameter adherent to the VCAM-1 functionalized surface. Most of the liposomes collapsed immediately after the interaction with VCAM-1 covered surface; at times, liposome in the process of deformation were observed next to a still intact one. As a parameter to follow the shape changes, the volume of the liposomes was estimated from the topography images by integration under the marked area, the volume between the liposome surface and the plane $\mathrm{z}=\mathrm{z}_{\min }$, where $\mathrm{z}_{\min }$ is the minimum value of height occurring in the marked area (Figure 3F and G). The difference in height between liposome and the sensor surface was measured and integrated over the $110 \mathrm{~nm}$ high liposome (non-collapsed liposome marked with red for measurement, Figure 3F) and over the collapsed liposome (marked with red for measurement, Figure 3G). The estimated volume of the $110 \mathrm{~nm}$ 
high liposome is $0.00177 \mu \mathrm{m}^{3}$, while the estimated volume of the collapsed liposome is 0.00179 $\mu^{3}$. Since the volume of a liposome after collapsing is not expected to change, as the same amount of liquid is present, only rearranged as a film instead of a vesicle, these measurements indicated that the image in Figure 4D portrays a collapsed and a non-collapsed liposome. The expected volume of a liposome can also be calculated for a given diameter. Using the volume formula of a sphere $\left(4 \pi \mathrm{R}^{3} / 3\right)$ we calculated that a $150 \mathrm{~nm}$ in diameter spherical liposome would have the same approximate volume $\left(0.00176 \mu^{3}\right)$ an indication that the topographical details on the sensor's surface represent the investigated liposomes. In contrast, no interaction with BSA covered surface could be detected (Figure 4E) confirming that the specific binding of TSL is a prerequisite for their deformation and potential cargo release.

\subsection{Upon binding, Vp-TSL release their content on the surface of activated EC}

A prerequisite for a suitable high activity at target sites is the destabilization of the liposomes after binding to the target cells and the resulting release the encapsulated cargo. To check whether this property applies to our liposome preparations we performed experiments to determine the kinetics of calcein release from $10 \mu \mathrm{M}$ TSL-1 incubated with quiescent or TNF- $\alpha$ activated EC (slightly fixed in $1 \%$ paraformaldehyde) for 1 to 24 hours at $37^{\circ} \mathrm{C}$ under static and flow conditions. Under static conditions, the fluorescence measurements indicated that Vp-TSL upon binding to activated EC released (at all times studied) the highest amount of encapsulated calcein having values of $\sim 15 \%$ and $\sim 74 \%$ after 1 and $24 \mathrm{~h}$, respectively (Figure $4 \mathrm{~A}$ and $\mathrm{B}$ ). In contrast, control liposomes (Vp-CL) released only $6 \%$ of the encapsulated marker after $24 \mathrm{~h}$ of incubation with activated EC. 
In flow conditions (flow chamber experiments), the release of calcein was determined upon incubation of non-targeted and targeted Vp-TSL with VCAM-1 Fc chimera or with quiescent or TNF $\alpha$-activated EC placed on glass slides and incorporated into a parallel plate flow chamber. The flow medium, (PBS, pH 7.4) was driven at a shear rate of $\sim 200 \mathrm{~s}-1$ and the fluorescence of calcein release was determined at different time points for 24 hours. Whereas Vp-TSL released nearly $80 \%$ of their payload at 24 hours after binding to immobilized VCAM1, only $60 \%$ has been released by TSL lacking the homing peptide (Figure 4C). A similar difference was also detected in the experiments in which the release of calcein upon binding of Vp-TSL to activated and non-activated EC were compared; in the latter case, the release rate was significantly lower compared to activated EC (Figure 4D).

\subsection{Teijin is released after interaction of Vp-TSL-Tj with activated EC and inhibit monocyte adhesion}

Teijin compound 1 was selected as pharmacological tool to search for the interference with the local inflammatory reactions in atherosclerosis. The CCR2 expression by EC and human PBMC was assessed by flow cytometry as outlined in the Supplementary Figure 2.

Furthermore, the hydrophilic properties of Teijin and its solubility in water are characteristics that should assure a comparable release as shown above for calcein. The encapsulation efficiency of Teijin into TSL was determined by HPLC after separation of entrapped from free Teijin; the calculated entrapment was about $75 \mu \mathrm{g}$ Teijin/ $\mu$ mol liposomes.

\subsubsection{Teijin is released from Vp-TSL-Tj after binding to activated EC surface}


The release characteristics of Teijin fromVp-TSL-Tj were in line with those obtained with the marker molecule, calcein, indicating a higher release in comparison with the amount liberated from conventional $\mathrm{Vp}-\mathrm{CL}-\mathrm{Tj}$. The concentration of Teijin released from different types of liposomes after their incubation with activated EC was determined by HPLC and the release was calculated in relation to the initial concentration of Teijin entrapped into liposomes (before incubation with EC). Upon incubation with activated EC, the highest release of Teijin was obtained in the case of Vp-TSL-Tj where the calculated values were $\sim 43 \%$ after 1 hour and more than $78 \%$ after 6 hours of incubation, while for non-targeted TSL (TSL-Tj) and targeted CL (Vp-CL-Tj) the figures obtained were $\sim 30 \%$ and $\sim 7 \%$ after 6 hours, respectively (Figure 5).

3.5.2. Vp-TSL-Tj inhibit monocyte adhesion and transmigration through activated EC monolayer

The adhesion of monocytes to activated EC was inhibited by $\sim 45 \%$ in the presence of free Teijin at $100 \mu \mathrm{g} / \mathrm{ml}$ concentration (Figure 6A). In the case of targeted TSL, the concentration of 100 $\mu \mathrm{g} / \mathrm{ml}$ Teijin incorporated into Vp-TSL-Tj determined the maximal inhibition of monocyte adhesion of $\sim 28 \%$; a further increase in the concentration does not enhance the effect. However, the inhibition by TSL-Tj or Vp-CL-Tj was significantly lower (13\% and 7\%, respectively). The efficacy of chemokine receptor inhibition by $\mathrm{Vp}$-TSL-Tj on transmigration of human monocytes through human activated EC was assessed by the classical transmigration assay using the Boyden chambers. The TNF- $\alpha$ activated EC seeded on the insert's filter were incubated with $100 \mu \mathrm{g} / \mathrm{ml}$ of either free Teijin or Vp-TSL-Tj, TSL-Tj and Vp-CL-Tj in the presence of calcein- 
AM labelled monocytes. In all experimental conditions, the percent inhibition of monocyte transmigration in the presence of Teijin, either free or incorporated into different types of liposomes, was determined relative to control (monocytes incubated with activated EC in the absence of free or encapsulated Teijin) by measuring the fluorescence of the cells in the lower chamber and trapped to the filter. A significantly higher inhibition of monocyte transmigration was obtained for Vp-TSL-Tj $(\sim 53 \%)$ as compared to non-targeted TSL-Tj $(\sim 32 \%)$ and Vp-CL$\mathrm{Tj}(\sim 18 \%)$.

\subsection{Animal Studies}

The in vivo effect of Teijin and targeted liposomes were investigated on ApoE-/- mice. First, we searched whether Teijin is functional in Apo E-/- mice by following the effect of pre-treatment of monocyte with Teijin on their adhesion/infiltration into the aorta of mice. Second, we tested whether Vp-TSL recognizes and bind specifically to activated aortic EC in ApoE-/- mice. Further preliminary experiments following the effect of the Vp-TSL-Tj treatment of ApoE-/mice showed that atherosclerotic lesions area was significantly reduced in treated animals (data not shown).

3.6.1. Teijin inhibits monocyte/macrophage (RAW 264.7 cell line) infiltration into mouse aorta

The effect of Teijin on the adhesion of monocytes to EC was evaluated in situ by pre-incubation of RAW 264.7 cells with CCR2 antagonist and determining their adhesion and accumulation in 
the aortas of ApoE-/- mice using an IVIS imaging system. It was found that compared to the sizeable accumulation of fluorescently labelled monocytes in the aorta of ApoE-/- mice, preincubation with Teijin decreased notably the binding and the infiltration of monocytes into the aorta (Figure 7). No infiltration of monocytes was detected in the aorta of C57BL/6 mice in the absence or the presence of Teijin. The results indicated that Teijin operates as an inhibitor of monocytes adhesion/infiltration to the aorta of mice.

\subsubsection{Vp-TSL bind specifically to the aorta of ApoE -/- mice}

The binding of Vp-TSL to the aortas of ApoE-deficient and C57BL/6 mice was investigated after in situ administration of Rhodamine-PE labelled liposomes and extensive washing to remove non-bound liposomes. The aortic arches, thoracic and abdominal aortas were harvested and viewed by fluorescent optical imaging employing an IVIS system. The images were quantified for fluorescent radiant efficiency [fluorescence emission radiance per incident excitation intensity: $\left.\left(\mathrm{p} / \mathrm{sec} / \mathrm{cm}^{2} / \mathrm{sr}\right) /\left(\mu \mathrm{W} / \mathrm{cm}^{2}\right)\right]$ using region-of-interest (ROI) function of the Living Image 4.3.1. software. The results revealed a higher binding to the aorta of Apo E-/- mice for Vp-TSL (at a radiant efficiency of $6.25 \pm 0.74 \times 10^{8}$ ) as compared to non-targeted TSL whose binding was significantly lower $\left(4.75 \pm 0.25 \times 10^{8}\right)$; no binding to the aorta of control C57BL mice was detected (Figure 8).

\section{Discussion}


Despite of the great progress in developing new technologies for diagnosis and/or therapeutics, the cardiovascular diseases (CVD) remain the leading cause of morbidity and mortality in the world. The inflammatory process is a common early event in atherogenesis, characterized by the expression of a specific array of cell adhesion molecules and chemokines on the endothelium that attract particular immune cells (e.g. monocytes, lymphocytes) to the developing atherosclerotic plaque. ${ }^{1}$ Since chemokines are critically involved in the development of atherosclerosis, the design of a targeted delivery nanosystem able to carry inhibitors of chemokine/chemokine receptor interaction at the surface of "inflamed" endothelium is desirable. Such a delivery system may constitute a therapeutic option to achieve a reduction of monocytes accumulation into vascular wall and consequently, to slow down or to prevent the inflammatory process.

To design a drug carrier system which possesses vascular targetability combined with a triggered drug release at the target site, we adapted the principle of the target-sensitive liposomes (TSL) described by Leaf Huang and collaborators ${ }^{12,13,14,26,27,28}$ to PEGylation to guarantee long circulation in the blood. During atherosclerosis VCAM-1 has been shown to be over-expressed at the endothelial surfaces. ${ }^{4,24,25}$, therefore, the attachment of a peptide with high affinity for VCAM-1 to the distal ends of PEG present in liposomes bilayers endows specificity of TSL for activated EC. Previously, TSL obtained by stabilization of unsaturated DOPE into bilayers using antibodies or peptide derivatives of fatty acids (palmitic acid) were employed for the delivery of antiviral agents ${ }^{12-14,26-28}$ or streptokinase. ${ }^{29,30}$

We performed optimization studies by varying fractions of DOPA or the PEG-DSPE derivatives to establish the liposomes composition that best guarantee the stability of the targeted carrier system in the blood until they reach the molecular target on the surface of activated EC. Based 
on these results we chose the TSL having in composition $60 \mathrm{~mol} \%$ DOPE, $35 \mathrm{~mol} \%$ DOPA and $5 \mathrm{~mol} \%$ PEG to be employed for all further functional studies of targeted release, as this composition assure the best stability in serum and at storage. These liposomes were able to efficiently encapsulate Teijin compound 1, an antagonist of chemokine receptor CCR2 (Vp-TSLTj) and deliver specifically at the surface of activated EC. The results of the competitive binding assay experiments revealed that Vp-TSL bind selectively and specifically to activated EC. Furthermore, we could provide an insight into the behaviour of Vp-TSL after binding to the target VCAM-1 by biosensor or SPFM measurements. These suggest a flattering or fusion of the TSL at the target surface as a prerequisite for their deformation-triggered release of the entrapped compounds.

In addition, in vitro evaluation of the release of entrapped calcein from TSL incubated with immobilized rhVCAM-1 or EC under static or flow conditions revealed a higher amount of release in the case of Vp-TSL incubated with rhVCAM-1 coated slides. Similarly, activated EC under static or flow conditions revealed a higher amount of release in the case of Vp-TSL at all times studied that was in contrast with non-targeted TSL and conventional Vp-CL liposomes. Only one previous study investigated the effects of different amount of PEG-derived phospholipids on the activity of target-sensitive liposomes. ${ }^{31} \mathrm{Ng}$ et al showed that target-induced destabilization does not take place when the amounts of $\mathrm{PEG}_{2000}$-DOPE higher than $0.3 \mathrm{~mol} \%$ are used for TSL preparation. On the contrary, our Vp-TSL composed of 5 mol \% $\mathrm{PEG}_{2000^{-}}$ DSPE bound to activated EC and released the highest amount of encapsulated calcein with values of $\sim 15 \%$ and $\sim 74 \%$ after 1 and $24 \mathrm{~h}$, respectively in comparison with non-targeted TSL and conventional liposomes. The percent of Teijin released at the surface of activated EC from $\mathrm{Vp}-\mathrm{TSL}-\mathrm{Tj}$ was $\sim 45 \%$ at $1 \mathrm{~h}$ of incubation. A possible explanation for the difference between 
our results and previous study ${ }^{31}$ could be based on the different preparation of liposomes. $\mathrm{Ng}$ et al coupled an antibody targeting p-glycoproteins to $\mathrm{N}$-glutarylphosphatidylethanolamine (N-glutPE) to the liposomal surface. Thus, the antibody could be masked by the increasing amount of PEG, leading to a reduced binding to the target and consequently, a reduced triggering of the release. In contrast, coupling the homing device to the distal ends of PEG, as performed in our study, appears to be the favourable condition to obtain both, sufficient target accessibility and a triggered cargo release apart from acceptable storage stability. Our results on serum stability confirm that increasing concentration of PEG-DSPE decrease the serum induced calcein release. However, we observed a higher release after interaction with serum for TSL with 1 mol \% $\mathrm{PEG}_{2000}$-DSPE (1 hour of incubation with $20 \%$ and $50 \%$ serum determined $\sim 65 \%$ and $\sim 75 \%$ release) in comparison with a release below $10 \%$ for TSL with 1 mol \% $\mathrm{PEG}_{2000}$-DOPE in $10 \%$ and $75 \%$ serum, observed previously ${ }^{31}$.

The water-soluble CCR2 antagonist was encapsulated into the aqueous compartment of TSL at a concentration of $\sim 75 \mu \mathrm{g}$ Teijin/ $\mu \mathrm{mol}$ liposomes, as indicated by HPLC measurements. The functional effect of Teijin, free or entrapped into liposomes (Vp-TSL-Tj) on monocyte adhesion and transmigration was followed in vitro, using TNF- $\alpha$ activated EC (aEC). We detected a higher reduction of human monocyte adhesion to activated EC when the cells were incubated in the presence of Vp-TSL-Tj in comparison with aEC incubated with TSL-Tj or Vp-CL-Tj. These data are indicative of the specificity of $\mathrm{Vp}-\mathrm{TSL}-\mathrm{Tj}$ and the release at the target surface of a higher amount of Teijin as compared to non-targeted TSL and Vp-CL-Tj. The inhibition of monocyte adhesion induced by cell treatment with free Teijin is higher than using any liposomal system entrapping Teijin. This finding is clearly related to the lower and slower Teijin release from the liposomes compared to the free drug, which is directly available. However, these 
experimental conditions neglect the physiological situation of blood flow and the potential benefit of the sustained local release of the drug from the liposomes. In the case of monocyte transmigration through aEC, a process that was followed for 16 hours, Vp-TSL-Tj generated an inhibition of transmigration that was superior to that obtained with same amount of free Teijin or TSL-Tj or Vp-CL-Tj. This result indicates that Vp-TSL-Tj besides releasing Teijin, also bind and block VCAM-1 that is actively involved in monocyte transmigration ${ }^{32}$, thus augmenting the percent inhibition of monocyte transmigration.

To test whether the Vp-TSL are functional in vivo we employed the ApoE-/- mice. Previous studies showed that VCAM-1 is overexpressed on EC covering the developing atherosclerotic plaque. ${ }^{33}$ We found that in comparison with non-targeted liposomes, Vp-TSL bind specifically and to a high extent to the aorta of ApoE-deficient mice, recommending VCAM-1 as a reliable target for liposomes carrying chemokine receptor antagonists. In addition, we tested whether the CCR2 antagonist is functional in mice by following the effect of Teijin on mouse monocyte/macrophage (RAW 264.7 cell line) adhesion/infiltration into the aorta of ApoEdeficient mice in situ. The results of these experiments revealed that pre-treatment of monocytes with Teijin inhibited monocytes adhesion/infiltration into the aorta indicating that locally delivered CCR2 antagonist could function in reducing the monocyte infiltration into atherosclerotic plaque.

\section{Conclusion}

The newly developed VCAM-1 directed PEGylated target-sensitive liposomes encapsulating Teijin, a CCR2 antagonist bind specifically to the surface of activated EC, release the entrapped 
drug and are functional in inhibiting the adhesion and transmigration of monocytes to/through activated EC. This study is the first evidence that a compound that interferes with chemokine/chemokine receptor interactions can be efficiently incorporated into designed liposomes for specific targeting of activated vascular endothelium. These novel findings hold great promises in reducing the chemokine-dependent inflammatory processes in many inflammation-related diseases, including atherosclerosis.

\section{Supporting information available}

The protocol for isolation of monocytes from human peripheral blood, the table containing the lipid composition of various formulations of target-sensitive liposomes (TSL) and the flow cytometry data on the expression of VCAM-1 and CCR2 on endothelial cells and of CCR2 on human monocytes are provided. This material is available free of charge via the Internet at http://pubs.acs.org.

\section{Notes}

The authors declare no competing financial interest.

Acknowledgments. The authors are indebted to Gabriela Mesca (technical assistance) and Emanuel Dragan (for assistance with animal experiments). This work was supported by 
UEFISCDI, Romania, Swiss National Science Foundation grant 31NM30-136033 (L.B.), project acronym NANODIATER under the frame of EuroNanoMed funded under the ERA-NET scheme of the Seventh Framework Programme of the European Commission.

\section{References}

1. Manduteanu, I., Simionescu, M. J. Cell. Mol. Med. 2012, 16(9)1978-1990.

2. Rot, A.; von Andrian U.H. Annu. Rev. Immunol. 2004, 22, 891-928.

3. Proudfoot, A.E.I.; Power, C.A.; Schwarz M.K. Expert Opin Investig Drugs 2010, 19, 345355.

4. Voinea, M.; Manduteanu, I.; Dragomir, E.; Capraru, M.; Simionescu, M. Pharm. Res. 2005, $22,1906-1917$.

5. Gosk, S. ; Moos, T.; Gottstein, C.; Bendas, G. Biochim. Biophys. Acta. 2008, 1778, 854-863.

6. Hua, S.; Chang, H.-I.; Davies, N.M.; Cabot, P.J. J. Liposome Res. 2011, 21, 95-105.

7. Voinea, M.; Dragomir, E.; Manduteanu, I.; Simionescu, M. Vascul. Pharmacol. 2002, 39, 1320.

8. Kessner, S.; Krause, A.; Rothe, U.; Bendas G. Biochim. Biophys. Acta. 2001,1514, 177-190.

9. Muro, S. J. Control. Release. 2012, 164, 125-137. 
10. Swaminathan, J.; Ehrhardt, C. Expert. Opin. Drug. Deliv. 2012, 9, 1489-1503.

11. Kumar, P.; Gulbake, A.; Jain, S.K. Crit. Rev. Ther. Drug. Carrier. Syst. 2012, 29, 355-419.

12. Ho, R.J.; Rouse, B.T.; Huang, L. Biochem. Biophys. Res. Commun. 1986,13, 931-937.

13. Ho, R.J.; Rouse, B.T.; Huang, L. Biochemistry 1986, 25, 5500-5506.

14. Ho, R.J.; Rouse, B.T.; Huang, L. J. Biol. Chem. 1987, 262, 13973-13978.

15. Sullivan, S.M.; Connor, J.; Huang, L. Med. Res. Rev. 1986, 6, 171-195.

16. Paternostre, M.; Ollivan, M.; Bolard, J. In Manual on Membrane Lipids; Prasad, R., Ed.; Springer-Verlag: Berlin, 1996; p 218-226.

17. Kelly, K.A.; Nahrendorf, M.; Yu, A.M.; Reynolds, F.; Weissleder, R. Mol. Imaging. Biol. 2006, 8, 201-207.

18. Martin, F.J.; Papahadjopoulos, D. J Biol Chem. 1982, 257, 286-288.

19. Edgell, C. J. S.; McDonald, C. C.; Graham, J. B. Proc. Natl. Acad. Sci. 1983, 80, 3734-3737.

20. Schlesinger, M.; Simonis, D.; Schmitz, P.; Fritzsche, J.; Bendas, G. Thromb. Haemost. 2009, $102,816-822$.

21. Manduteanu, I.; Pirvulescu, M.; Gan, A.M.; Stan, D.; Simion, V.; Dragomir, E.; Calin, M.; Manea, A.; Simionescu, M. Biochem. Biophys. Res. Commun. 2010, 391,1443-1448.

22. (Pasceri V et al., Modulation of Vascular Inflammation In Vitro and In Vivo by Peroxisome Proliferator-Activated Receptor-g Activators, Circulation. 2000;101:235-238).

23. Fang WB, Jokar I, Zou A, Lambert D, Dendukuri P, Cheng N. J. Biol. Chem. 2012, 287, 36593-36608.

24. Koning, G.A.; Schiffelers, R.M.; Storm, G. Endothelium 2002, 9, 161-171

25. Li, H.; Cybulsky, M.I.; Gimbrone, M.A.Jr.; Libby, P. Arterioscler. Thromb. 1993, 13, 197204. 
26. Ho, R.J.; Rouse, B.T.; Huang, L. J. Biol. Chem. 1987, 262, 13979-13984.

27. Ho, R.J.; Ting-Beall, H.P.; Rouse, B.T.; Huang, L. Biochemistry 1988, 27, 500-506.

28. Pinnaduwage, P.; Huang, L. Biochemistry 1992, 31, 2850-2855.

29. Vaidya, B.; Nayak, M.K.; Dash, D.; Agrawal, G.P.; Vyas, S.P. Int. J. Pharm. 2011, 403, 254-261.

30. Vaidya, B.; Nayak, M.K.; Dash, D.; Agrawal, G.P.; Vyas, S.P. Drug. Deliv. 2014, DOI: 10.3109/10717544.2014.916770

31. Ng, K.; Zhao, L.; Liu, Y.; Mahapatro, M. Int. J. Pharm. 2000, 193, 157-166.

32. Takahashi, M.; Ikeda, U.; Masuyama, J.; Kitagawa, S.; Kasahara, T.; Saito, M.; Kano, S; Shimada, K. Atherosclerosis 1994,108,73-81.

33. Cybulsky, M.I.; Iiyama, K.; Li, H.; Zhu, S.; Chen, M.; Iiyama, M.; Davis, V.; GutierezRamos, J.C.; Connely, P.W.; Milstone, D.S. J. Clin. Invest. 2001, 107, 1255-1262. 